This item was submitted to Loughborough's Institutional Repository (https://dspace.lboro.ac.uk/) by the author and is made available under the following Creative Commons Licence conditions.

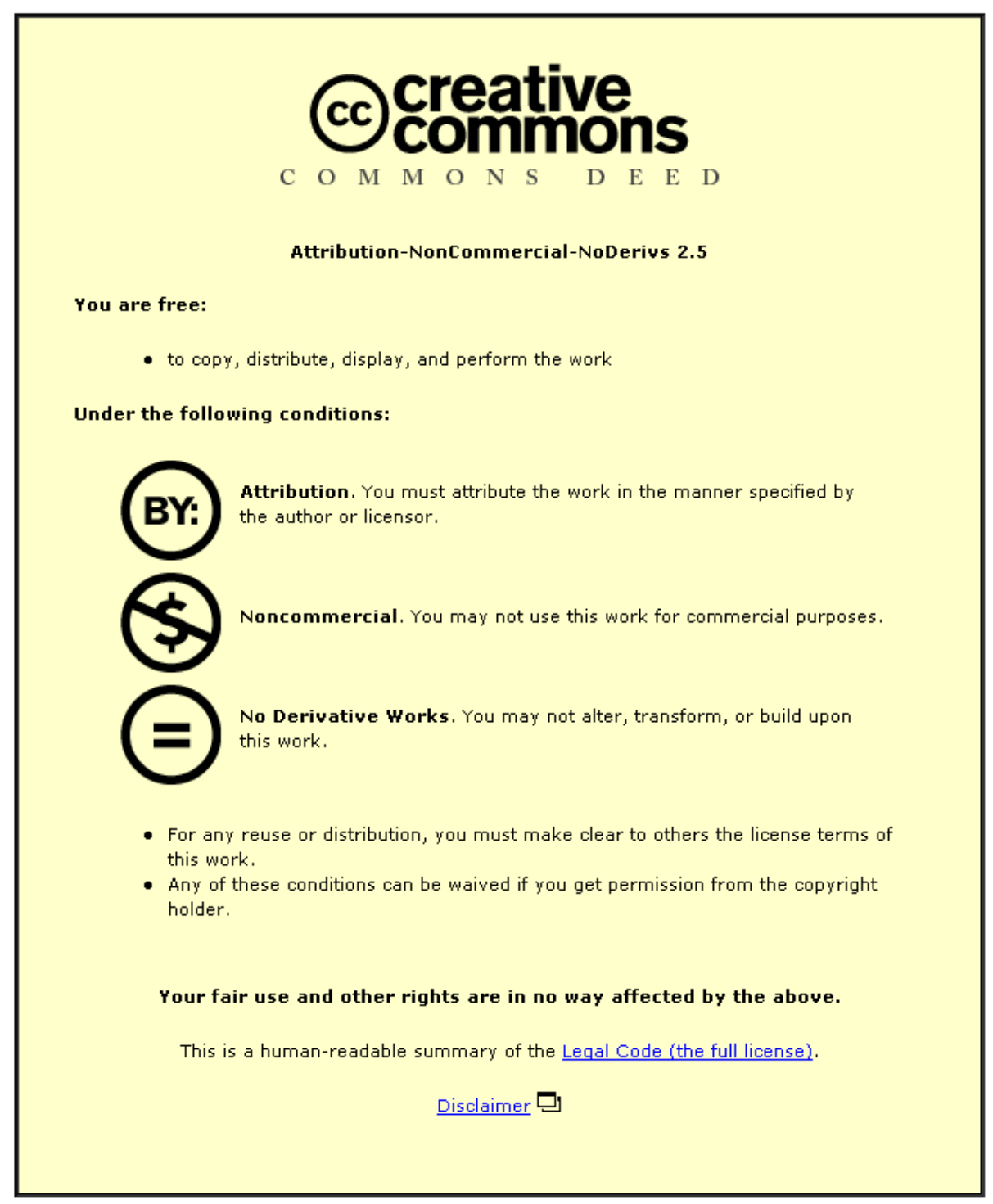

For the full text of this licence, please go to: http://creativecommons.org/licenses/by-nc-nd/2.5/ 


\title{
High accuracy tightly-coupled integrity monitoring algorithm for map-matching
}

\author{
Liang $\mathrm{Li}^{* \mathrm{a}, \mathrm{b}}$, Mohammed Quddus*b, Lin Zhao ${ }^{\mathrm{a}}$ \\ ${ }^{\mathrm{a}}$ College of Automation \\ Harbin Engineering University \\ Harbin 150001, P. R. China \\ Email: liliang@hrbeu.edu.cn \\ ${ }^{\mathrm{b}}$ Transport Studies Group \\ Department of Civil and Building Engineering \\ Loughborough University \\ Leicestershire LE11 3TU, UK \\ Email:m.a.quddus@lboro.ac.uk \\ * Corresponding Author
}

\begin{abstract}
A map-matching algorithm employs data from Global Positioning System (GPS), a Geographic Information System (GIS)-based road map and other sensors to first identify the correct link on which a vehicle travels and then to determine the physical location of the vehicle on the link. Due to uncertainties associated with the raw measurements from GPS/other sensors, the road map and the related methods, it is essential to monitor the integrity of map-matching results, especially for safety and mission-critical intelligent transport systems such as positioning and navigation of autonomous and semi-autonomous vehicles. Current integrity methods for map-matching are inadequate and unreliable as they fail to satisfy the integrity requirement due mainly to incorrect treatment of all the related uncertainties simultaneously. The aim of this paper is therefore to develop a new tightlycoupled integrity monitoring method for map-matching by properly treating the uncertainties from all sources concurrently. In this method, the raw measurements from GPS, low-cost Dead-Reckoning (DR) sensors and Digital Elevation Model (DEM) are first integrated using an extended Kalman Filter to continuously obtain better position fixes. A weight-based topological map-matching process is then developed to map-match position fixes on to the road map. The accuracy of the map-matching process is enhanced by employing a range of network features such as grade separation, traffic flow directions and the geometry of road link. The Receiver Autonomous Integrity Monitoring (RAIM) technique, which has been successfully applied to monitor the integrity of aircraft navigation, is modified and enhanced so as to apply it to monitor the quality of map-matching. In the enhanced RAIM method, two modifications are made: (1) a variable false alarm rate (as opposed to a constant false alarm rate) is considered to improve the fault detection performance in selecting the links, especially near junctions. (2) a sigma inflation for a non-Gaussian distribution of measurement noises is applied for the purpose of satisfying the integrity risk requirement.

The implementation and validation of the enhanced RAIM method is accomplished by utilising the required navigation performance parameters (in terms of accuracy, integrity and availability) of safety and mission-critical intelligent transport systems. The required data were collected from Nottingham and central London. In terms of map-matching, the results suggest that the developed map-matching method is capable of identifying at least $97.7 \%$ of the links correctly in the case of frequent GPS outages. In terms of integrity, the enhanced
\end{abstract}


RAIM method provides better the fault detection performance relative to the traditional RAIM.

Keywords: land vehicle navigation; map-matching; integrity; RAIM; extended Kalman filter.

\section{Introduction}

Land vehicle navigation has become an integral part of many location-based intelligent transport systems (ITS) over the last decade. In order to satisfy the positioning and navigation requirement of more stringent ITS services such as accident and emergency response (e.g. eCall in Europe), various collision avoidance services, positioning and navigation of intelligent and autonomous vehicles, transport of hazardous material and distance-based road user charging, a land vehicle navigation system should provide high reliability navigation and positioning solutions at low-cost. GPS has widely been utilised as the main positioning technology for land vehicle navigation. This is because GPS offers a number of unique advantages: it is free-of-charge for users and can provide 24-hour, all-weather and independent high accuracy 3-D positioning and timing solution. GPS however suffers from the line-of-sight problem, especially in the low satellite visibility environment such as urban canyons, tunnels, densely tree-lined streets and near fly-overs (Quddus and Zheng 2011). In those relatively harsh operational environments, stand-alone GPS cannot achieve the required navigation performance for many location-based ITS (Feng and Ochieng 2007). To compensate this deficiency, GPS has to be integrated with other sensors, such as DeadReckoning (DR) and Geographic Information System (GIS) based road map to provide horizontal positioning supplement and a Digital Elevation Model (DEM) to reduce the error in the vertical direction. Integration of data from various sources is primarily carried out in two modes (Bhatti 2007): loosely-coupled and tightly-coupled. It has been proven that a tightly-coupled system provides advantages over a loosely-coupled system, especially under the conditions of less than four satellites in-view or in the case of poor satellite geometry even if the number of visible satellites is more than four (Bhatti 2007; Quddus and Zheng 2011). This kind of scenarios is more frequent in urban operational environments.

Positioning solutions can further be enhanced by employing a map-matching algorithm that identifies the correct link (among the candidate links) on which a vehicle travels and determines the location of the vehicle on that link (White et al., 2000; Chen et al., 2005). Although a significant progress has made in the area of map-matching over the past decade, current map-matching methods still struggle to satisfy the required navigation performance, especially in harsh operational environments such as grade separation or interchanges, flyovers, complex roundabouts (e.g. Quddus et al., 2006; Feng and Ochieng 2007). The possible reasons are: (1) quality of initial position fixes from a navigation system to a map-matching algorithm as discussed earlier (2) lack of additional node and link attributes (e.g. curvature, grade separation, direction) in the map data and (3) lack of incorrect link detection ability from integrity monitoring. This paper aims to address these issues simultaneously by developing a tightly-coupled integrity monitoring algorithm for map-matching.

In terms of map data limitation as stated above, some recent GIS road databases now contain such network features as a result of recent advancement in data acquisition technologies. One example is the UK's Integrated Transport Network database which has a number of such features: whether a junction is grade separated and the direction of traffic flows. The inclusion of such features in map-matching may further enhance the performance of land vehicle navigation systems. Once map-matching results are obtained through a tightly-coupled navigation system aided by a map-matching algorithm, it is important to monitor the quality (i.e. level of confidence) of position solutions, especially for some safety 
and mission-critical land vehicle navigation. The method is known as integrity monitoring (Quddus et al., 2006). Integrity includes the ability of a navigation system to provide valid and timely warnings to users when the misleading information from the system is detected under the pre-set quantities which are decided by the probabilities of false alarm and missed detection (Ochieng et al., 2003). Unlike aircraft navigation in which the quality of positioning solution is the only concern, there are two types of failure in map-matching: (a) correct link identification and (b) location determination. Existing map-matching integrity methods focus either on the failure in the link identification (Quddus et al., 2006; Velaga et al., 2010) or the failure in the location estimation (Feng and Ochieng 2007; Velaga et al., 2010). Quddus et al. (2006) employ a fuzzy logic approach to detect mismatch in the link identification. Feng and Ochieng (2007) use a Receiver Autonomous Integrity Monitoring (RAIM ${ }^{1}$ ) to detect failure in the position estimation. Velaga et al. (2010) utilise a sequential approach to detect failures (i) in GPS raw measurements using the RAIM method (ii) in the link identification using a fuzzy logic approach. None of these above methods consider a range of inherent limitations with the application of RAIM method in the case of map-matching. The limitations are:

(i) Traditional RAIM (i.e. a snapshot method) is primarily based on the two components: fault detection and protection levels (horizontal and vertical) associated with the targeted integrity risk. While calculating the Horizontal Protection Level $\left(\mathrm{HPL}^{2}\right)$, most of the existing studies assumed that the error perfectly follows the Gaussian distribution. The assumption may however be inappropriate for the urban environment due to the severe multipath effect or lack of visible satellites. Therefore, a non-perfect Gaussian error distribution needs to be considered and the HPL needs to be properly calculated so as to satisfy the integrity risk requirement. In such a case, the measurement fault and the measurement bias may become correlated with each other (Blanch et al., 2007; Ober 2003). The correlation may increase the test statistic and decrease the position error. This condition may create a false alarm in the integrity monitoring. It is also likely that measurement noises cause the test statistic to decrease and the position error to increase. This condition may create a case of missed detection in the integrity monitoring. Some methods are proposed in the literature that can be applied to deal with the effects of measurement fault and measurement noises in the calculation of HPL such as the autonomous integrity monitoring extrapolation (AIME) proposed by Diesel et al. (1995) and the solution separation method proposed by Lee and O’Laughlin (2000).

(ii) In terms of fault detection, most of the existing studies have focused on either the failure of raw measurements related to the integrated navigation system (Feng and Ochieng 2007; Velaga et al., 2010) or the failure in the link identification (Quddus et al., 2006; Velaga et al., 2010) in map-matching. Both failures need to be considered simultaneously because a misleading positioning result may lead to link mis-identification in the map-matching process. Although a constant false alarm rate (CFAR) in the traditional RAIM has found to be suitable in detecting the positioning failure, a small-bias fault generated from the incorrect link identification may results in more missed detection cases. The CFAR based fault detection should not be utilised in map-matching.

This paper aims to address the above limitations and therefore, the objective of this paper is to develop a significantly enhanced RAIM for monitoring the positioning output of an integrated navigation system (GPS/DR/DEM) aided by a map-matching algorithm. An

\footnotetext{
${ }^{1}$ According to Parkinson et al. (1996), RAIM is termed as statistical consistency checks using redundant measurements. Two processes are related to RAIM. One is using protection levels to determine whether the condition is executable for a RAIM calculation. After that the test statistic is used to detect whether there is a fault in the measurements.

${ }^{2}$ HPL is the test statistic for the process of RAIM (i.e. RAIM availability). HPL should be less than the required horizontal alert limit to satisfy the RAIM availability requirement.
} 
extended Kalman Filter is employed to integrate data from GPS, DR and DEM in the tightlycoupled mode. The output of GPS/DR/DEM is then matched onto the Integrated Transport Network data using a topological map-matching algorithm that employs different node and link features such as road curvature, grade separation and traffic flow directions. Traditional RAIM algorithm is then enhanced in two ways: (1) the use of variable false alarm rate (VFAR) so as to improve the fault detection performance with the hypothesis that a fault may arise either from the raw measurements associated with the GPS/DR/DEM or the link identification in the map-matching process and (2) the consideration of non-Gaussian distribution for the measurement noises with the aid of a sigma inflation algorithm so as to satisfy the integrity risk requirement.

The reminder of the paper is organised as follows: first, the observation and state models for the tightly-coupled map-matching aided GPS/DR/DEM integrated navigation system is presented. It is then followed by a description of the enhanced RAIM algorithm including the formulation of sigma inflation based HPL for the targeted integrity risk requirement and the fault detection algorithm. The experimental results and the associated performance of the developed methods are then presented. The paper ends with conclusions and future research directions.

\section{The Observation Model for GPS/DR/DEM Aided by Map-Matching}

As discussed, a tightly-coupled system fuses data in the measurement domain from different systems/sensors. This section briefly discusses the raw measurements from each of the three components (i.e. GPS, DR and DEM) of the integrated positioning system. After obtaining the initial position estimation, a map-matching algorithm is introduced so as to increase the number of redundant measurements in the observation model.

\subsection{Raw measurements from GPS}

GPS can provide two kinds of raw measurement: carrier phase and pseudorange (Misra and Enge 2001). Since the initial ambiguity resolution for the carrier phase single point positioning is a time-consuming process, pseudorange measurements are therefore employed in this research. The linear model of pseudorange measurements in the World Geodetic System (WGS) 84 can be expressed as (Parkinson et al., 1996):

$$
\Delta \boldsymbol{\rho}_{k}=\boldsymbol{H}_{k}^{\mathrm{WGS} 84} \boldsymbol{X}_{k}^{\mathrm{WGS} 84}+\boldsymbol{\varepsilon}_{k}
$$

where $\Delta \boldsymbol{\rho}$ is the $n \times 1$ vector of the differences between the observed and corrected range measurements, $n$ is the number of available satellites, $\boldsymbol{H}$ is the $n \times 4$ observation matrix, $\boldsymbol{X}$ is the four dimensional vector $\left(\Delta x_{k}, \Delta y_{k}, \Delta z_{k}, \Delta t_{k}\right)$ of the corrections to the unknown user position parameters and the user receiver clock-bias (in metres). The term $\varepsilon$ represents the measurement noise vector $n \times 1$ with the distribution satisfying $\varepsilon \sim N\left(0, \sigma_{\mathrm{GPS}}^{2}\right)$ in which $\sigma_{\mathrm{GPS}}$ is the standard deviation of the pseudorange noises. The subscript $k$ is the index for the $k$ th epoch. Since the raw measurements from the other sensors (i.e. DR and DEM) are based on the local-frame coordinate, it is necessary to transform the WGS84 based GPS measurements into the local-frame. Given the origin of the local frame, the transformation matrices $\boldsymbol{T}_{\text {ref }}$ can be obtained such that: 


$$
\left[\begin{array}{c}
\Delta \varphi_{k} \\
\Delta \lambda_{k} \\
\Delta h_{k}
\end{array}\right]=\boldsymbol{T}_{\text {ref }}\left[\begin{array}{c}
\Delta x_{k} \\
\Delta y_{k} \\
\Delta z_{k}
\end{array}\right]
$$

where $\Delta \varphi_{k}, \Delta \lambda_{k}, \Delta h_{k}$ represent the east, north and height of the user coordinate corrections in the local-frame and can be expressed as:

$$
\left.\begin{array}{l}
\Delta \varphi_{k}=\varphi_{k}-\varphi_{k-1} \\
\Delta \lambda_{k}=\lambda_{k}-\lambda_{k-1} \\
\Delta h_{k}=h_{k}-h_{k-1}
\end{array}\right\}
$$

Substitute Eq.(2) into Eq.(1), the measurement model in the local frame can be expressed as:

$$
\Delta \boldsymbol{\rho}_{k}=\boldsymbol{H}_{k}^{\text {enu }} \boldsymbol{X}_{k}^{\text {enu }}+\boldsymbol{\varepsilon}_{k}
$$

where $\boldsymbol{X}_{k}^{\text {enu }}$ is the corrections to the unknown parameters $\left(\Delta \varphi_{k}, \Delta \lambda_{k}, \Delta h_{k}, \Delta t_{k}\right)$ and $\boldsymbol{H}^{\mathrm{enu}}$ is the observation matrices in the local frame and this can be achieved as:

$$
\boldsymbol{H}^{\text {enu }}=\left[\begin{array}{ll}
\boldsymbol{H}_{n \times 3}^{\text {WGS84 }} \boldsymbol{T}_{\text {ref }}^{-1} & -\mathbf{1}_{n \times 1}
\end{array}\right]
$$

\subsection{Raw measurements from $D R$}

In DR sensors, a one-axis gyroscope is used as a heading sensor to measure the angle difference relative to the local north direction and an odometer is used as a displacement sensor to measure the relative travel distance within a sampling interval. The raw measurements in the local frame can be expressed as:

$$
\left\{\begin{array}{l}
\theta_{k}=\arctan \left(\frac{v_{e, k}}{v_{n, k}}\right)+v_{\mathrm{DR} 4 \text { angle }, k} \\
d_{k}=\sqrt{\Delta \varphi_{k}^{2}+\Delta \lambda_{k}^{2}}+v_{\mathrm{DR} 4 \text { distance }, k}
\end{array}\right.
$$

where $\theta$ is the angular difference from the gyroscope and $d$ is the displacement from the odometer output. $v_{e}$ and $v_{n}$ are the speed in the east and north direction respectively. $v_{\mathrm{DR} \text { angle }}$ and $v_{\mathrm{DR} \text { 4distance }}$ are the corresponding measurements noises. It should be noted that the positioning error in DR sensors accumulates over time due to the inherent limitations in the inertial sensors (Parkinson et al., 1996). Therefore, the data from GPS are used to calibrate the data from DR so as to obtain better positioning accuracy.

\subsection{Raw measurements from DEM}

A DEM is a digital representation of ground surface topography or terrain providing height data. The measurements from the DEM can therefore be expressed as:

$$
\Delta H_{k}=\Delta h_{k}+v_{\mathrm{DEM}, k}
$$


in which $\Delta H_{k}=H_{k}-H_{k-1}$ is the difference of the DEM output between the adjacent epochs. $v_{\mathrm{DEM}}$ is the measurement noise from the DEM output.

\subsection{Augmentation from map-matching}

Using the raw measurements from GPS, DR, and DEM, the user position can continuously be obtained through the extended Kalman filter algorithm (discussed below). The positioning output of the filter is used to identify the correct link. Since road network data include not only the basic road geometry information (such as node coordinates and link ID) but also the traffic flow directions and the grade separation information, a weight-based topological map-matching algorithm developed by Velaga et al. (2010) is modified so that additional link and node features can be incorporated. In this algorithm, each of the candidate links is assigned a weight based on the connectivity between a candidate link and the previous identified link, the heading difference between the candidate link and the vehicle heading from the extended Kalman filter algorithm and the proximity between the GPS fix and the candidate link. The total weight score for each of the candidate links is calculated and the candidate link with the highest total weight score is chosen as the correct link for the position fix. TWS is expressed as follows:

$$
T W S_{i}=f(\text { connectivity }) \times\left[w_{i}^{\mathrm{pD}} \times f\left(P D_{\perp}\right)+w_{i}^{\mathrm{HD}} \times f(H D)\right]
$$

In which

$$
f(\text { connectivity })=\left\{\begin{array}{cc}
1 & \text { connected } \\
-1 & \text { else }
\end{array}, f\left(P D_{\perp}\right)=\left\{\begin{array}{cc}
1-\frac{\left|\Delta x_{\perp}\right|}{T H_{m m}} & \left|\Delta x_{\perp}\right| \leq T H_{m m} \\
-1 & \text { else }
\end{array}, f(H D)=\cos \Delta \theta\right.\right.
$$

where $f$ (connectivity), $f\left(P D_{\perp}\right)$ and $f(H D)$ represent the functions related to the connectivity, proximity (i.e. perpendicular distance) $\Delta x_{\perp}$ and heading difference $\Delta \theta$ respectively. $T H_{m m}$ is the threshold for the position error indicating the allowable maximum positioning error in the positioning data from the GPS/DR/DEM system. $w_{i}^{\mathrm{PD}}$ and $w_{i}^{\mathrm{HD}}$ are the weights for the proximity and heading difference and the condition to determine their values is: $w_{i}^{\mathrm{PD}}+w_{i}^{\mathrm{HD}}=1$. The values can be obtained either from an empirical analysis of a true input-output dataset or an optimal method proposed by Velaga et al. (2010). TWS ${ }_{i}$ is the total weight score for the ith candidate link.

It should be noted that the mapping of the first GPS fix on the newly identified link close to a junction is very important as the results of the subsequent position fixes largely depends on the correctness of the first map-matching. This is because link length of the selected link is employed to map-match the subsequent fixes on the same link (Velaga et al., 2010). The map-matching at a junction is however challenging because there are many candidate links from which the correct link needs to be identified and therefore, it is likely that incorrect link identification may create a small-bias fault, i.e. $\mathrm{A}_{\mathrm{T}} \mathrm{A}_{\mathrm{PM}}$. in Fig. 1 in which $\mathrm{A}$ is the initial user position solution, $A_{T}$ is the true user position, $A_{P M}$ and $A_{P Q}$ are the map-matched user position on the incorrect link PM and the correct link PQ respectively. In such a case, traditional RAIM would be inadequate to detect the false link identification as CFAR has its limitation in dealing with small-bias faults due to many candidate links. An enhanced fault detection method is needed to address this issue. 


\section{INSERT FIGURE 1 HERE}

After the identification of the correct link, the next step of the map-matching algorithm is to estimate the vehicle location on that link. This is briefly explained below:

Assume that $\mathrm{P}\left(\varphi_{b}, \lambda_{b}\right)$ and $\mathrm{Q}\left(\varphi_{e}, \lambda_{e}\right)$ denote start-node coordinates and end-node coordinates of the correct link PQ, a constraint equation for the map-matched user position fix can be developed as follows:

$$
\lambda_{k}=\frac{\lambda_{b}-\lambda_{e}}{\varphi_{b}-\varphi_{e}}\left(\varphi_{k}-\varphi_{e}\right)+\lambda_{e}+v_{\text {map }, k}
$$

In which $v_{\text {map }}$ is the map error in the map data. Equation (8) can be rearranged as follows: To be compatible with the system observation model that is presented in the next section, the constraint equation can be rewritten as:

$$
\left[\left(\varphi_{b}-\varphi_{e}\right) \lambda_{e}-\left(\lambda_{b}-\lambda_{e}\right) \varphi_{e}-\left(\lambda_{e}-\lambda_{b}\right) \varphi_{k-1}-\left(\varphi_{b}-\varphi_{e}\right) \lambda_{k-1}\right]=\left[\begin{array}{ll}
\lambda_{e}-\lambda_{b} & \varphi_{b}-\varphi_{e}
\end{array}\right]\left[\begin{array}{c}
\Delta \varphi_{k} \\
\Delta \lambda_{k}
\end{array}\right]+v_{\text {maa }, k}
$$

\subsection{The observation Model}

The observation model for the GPS/DR/DEM aided by the map-matching process can be obtained by combining Eq.(3), (5), (6) and (10). This can be expressed as follows:

$$
\boldsymbol{y}_{k}=f\left(\boldsymbol{X}_{k}^{\mathrm{enu}}\right)+\boldsymbol{v}_{k}
$$

In which

$$
\begin{aligned}
& \boldsymbol{Y}_{k}=\left[\begin{array}{c}
\Delta \rho_{1, k} \\
\vdots \\
\Delta \rho_{n, k} \\
\theta_{k} \\
d_{k} \\
\Delta H_{k} \\
\left(\varphi_{b}-\varphi_{e}\right)\left(\lambda_{e}-\lambda_{k-1}\right)-\left(\lambda_{b}-\lambda_{e}\right)\left(\varphi_{e}-\varphi_{k-1}\right)
\end{array}\right], v_{k}=\left[\begin{array}{c}
\varepsilon_{1, k} \\
\vdots \\
\varepsilon_{n, k} \\
v_{\mathrm{DR} 4 \text { angle }, k} \\
v_{\mathrm{DR} 4 \text { distance }, k} \\
v_{\mathrm{DEM}} \\
v_{\text {map }}
\end{array}\right] \\
& f\left(\boldsymbol{X}_{k}^{\mathrm{enu}}\right)=\left[\begin{array}{c}
\boldsymbol{H}_{1,1}^{\mathrm{enu}} \Delta \varphi_{k}+\boldsymbol{H}_{1,2}^{\mathrm{enu}} \Delta \lambda_{k}+\boldsymbol{H}_{1,3}^{\mathrm{enu}} \Delta h_{k}-\Delta t_{k} \\
\vdots \\
\boldsymbol{H}_{n, 1}^{\mathrm{enu}} \Delta \varphi_{k}+\boldsymbol{H}_{n, 2}^{\mathrm{enu}} \Delta \lambda_{k}+\boldsymbol{H}_{n, 3}^{\mathrm{enu}} \Delta h_{k}-\Delta t_{k} \\
\arctan \left(\frac{v_{e, k}}{v_{n, k}}\right) \\
\sqrt{\Delta \varphi_{k}^{2}+\Delta \lambda_{k}^{2}} \\
\Delta h_{k} \\
\left(\lambda_{e}-\lambda_{b}\right) \Delta \varphi_{k}+\left(\varphi_{b}-\varphi_{e}\right) \Delta \lambda_{k}
\end{array}\right]
\end{aligned}
$$

where $\boldsymbol{v}_{k}$ is a $(n+4) \times 1$ vector and represent the measurements error. 


\section{The State Model}

Due to the nonlinear nature of the observation model, an extended Kalman filter can be utilised to achieve the optimal state estimation. The position-velocity model that is in-line with the observation model is employed as the state model in which the user height correction and clock bias are modelled as the first-order Markov process:

$$
\left[\begin{array}{c}
\Delta \varphi_{k} \\
\Delta \lambda_{k} \\
\Delta h_{k} \\
v_{e, k} \\
v_{n, k} \\
\Delta t_{k}
\end{array}\right]=\left[\begin{array}{cccccc}
1 & 0 & 0 & T_{s} & 0 & 0 \\
0 & 1 & 0 & 0 & T_{s} & 0 \\
0 & 0 & 1+\frac{T_{s}}{\tau_{h}} & 0 & 0 & 0 \\
0 & 0 & 0 & 1 & 0 & 0 \\
0 & 0 & 0 & 0 & 1 & 0 \\
0 & 0 & 0 & 0 & 0 & 1+\frac{T_{s}}{\tau_{t}}
\end{array}\right]\left[\begin{array}{c}
\Delta \varphi_{k-1} \\
\Delta \lambda_{k-1} \\
\Delta h_{k-1} \\
v_{e, k-1} \\
v_{n, k-1} \\
\Delta t_{k}
\end{array}\right]+w_{k}
$$

In which the parameters $\tau_{h}$ and $\tau_{t}$ in transition matrix $\boldsymbol{\Phi}_{k \mid k-1}$ are the auto-correlation time constants for the first order Markov model, $\boldsymbol{w}_{k}$ is a $6 \times 1$ process noises vector, $T_{s}$ is the sampling interval, and the state vector $\boldsymbol{x}_{k}$ is

$$
\boldsymbol{x}_{k}=\left[\begin{array}{llllll}
\Delta \varphi_{k} & \Delta \lambda_{k} & \Delta h_{k} & v_{e, k} & v_{n, k} & \Delta t_{k}
\end{array}\right]^{\mathrm{T}}
$$

After setting up the initial state estimation and initial state estimation covariance, the map-matching aided filtering process can be implemented as follows:

$$
\left.\begin{array}{l}
\hat{\boldsymbol{x}}_{k \mid k-1}=\boldsymbol{\Phi}_{k \mid k-1} \boldsymbol{X}_{k-1} \\
\hat{\boldsymbol{y}}_{k \mid k-1}=f\left(\hat{\boldsymbol{x}}_{k \mid k-1}\right) \\
\boldsymbol{P}_{k \mid k-1}=\boldsymbol{\Phi}_{k \mid k-1} \boldsymbol{P}_{k-1} \boldsymbol{\Phi}_{k \mid k-1}^{\mathrm{T}}+\boldsymbol{Q}_{k-1} \\
\boldsymbol{K}_{k}=\boldsymbol{P}_{k \mid k-1} \boldsymbol{G}_{k}\left(\boldsymbol{G}_{k} \boldsymbol{P}_{k \mid k-1} \boldsymbol{G}_{k}^{\mathrm{T}}+\boldsymbol{R}_{k}\right)^{-1} \\
\boldsymbol{P}_{k}=\left(\boldsymbol{I}-\boldsymbol{K}_{k} \boldsymbol{G}_{k}\right) \boldsymbol{P}_{k \mid k-1} \\
\hat{\boldsymbol{x}}_{k}=\hat{\boldsymbol{x}}_{k \mid k-1}+\boldsymbol{K}_{k}\left(\boldsymbol{y}_{k}-\hat{\boldsymbol{y}}_{k \mid k-1}\right)
\end{array}\right\}
$$

in which 


$$
\boldsymbol{G}_{k}=\left[\begin{array}{cccccc}
\boldsymbol{H}_{11, k} & \boldsymbol{H}_{12, k} & \boldsymbol{H}_{13, k} & 0 & 0 & -1 \\
\vdots & \boldsymbol{H}_{n 2, k} & \boldsymbol{H}_{n 3, k} & 0 & 0 & -1 \\
\boldsymbol{H}_{n 1, k} & 0 & 0 & \frac{\hat{v}_{n, k \mid k-1}}{\hat{v}_{e, k \mid k-1}^{2}+v_{n, k \mid k-1}^{2}}-\frac{\hat{v}_{e, k}}{v_{e, k \mid k-1}^{2}+\hat{v}_{n, k \mid k-1}^{2}} & 0 \\
0 & \frac{\Delta \hat{\lambda}_{k \mid k-1}}{\Delta \hat{\varphi}_{k \mid k-1}} & 0 & 0 & 0 & 0 \\
\frac{\sqrt{\Delta \hat{\varphi}_{k \mid k-1}^{2}+\Delta \hat{\lambda}_{k \mid k-1}^{2}}}{\sqrt{\Delta \hat{\varphi}_{k \mid k-1}^{2}+\Delta \hat{\lambda}_{k \mid k-1}^{2}}} & 0 & 1 & 0 & 0 & 0 \\
0 & \varphi_{b}-\varphi_{e} & 0 & 0 & 0 & 0
\end{array}\right]
$$

It should be noted that Eq. (11) implies the observation model of the GPS/DR/DEM system aided by a map-matching algorithm. Since there is no information regarding the correct link on which a vehicle travels before map-matching, the last measurement from the observation model needs to be excluded so as to obtain the initial position solution from the GPS/DR/DEM system (i.e. Point A in Figure 1). The output of this GPS/DR/DEM needs to be fed into the link identification process of map-matching. Once the correct link is being identified, Eq. (13) i.e. the map-matching aided extended Kalman filter can be applied to obtain the final user position solution (i.e. Point B in Figure 1). In order to obtain the mapmatched point (i.e. Point $\mathrm{B}_{\mathrm{PQ}}$ in Figure 1), Point $\mathrm{B}$ is perpendicularly projected on the corrected link (i.e. link PQ in Figure 1). It is expected that the positioning accuracy of GPS/DR/DEM system can be improved as additional information from the map-matching is employed.

\section{Enhanced RAIM for Map-Matching}

As discussed, traditional RAIM algorithms may not be capable of detecting the incorrect link identification as a result of a failure in the map-matching process. As there are multiple candidate links, the user position on the wrong link can be regarded as a small-bias fault. In this case, fault detection performance should be more stringent and relate to the number of candidate links.

Figure 2 presents the flowchart of the developed enhanced RAIM algorithm in terms of required input and intended output. As can be seen, the algorithm takes input data from a number of sources: GPS/DR/DEM, map-matching and the uncertainties in the measurement errors. The output of the enhanced RAIM includes the HPL and fault detection that are subsequently used to alert the user about the quality of the map-matched position.

\section{INSERT FIGURE 2 HERE}

\subsection{HPL for GPS/DR/DEM/MM}

The protection level is defined as the upper bound on the condition that a derived total system error must not exceed without being detected for a given probability. Under the single-fault hypothesis, the total system error may cause by the likely faults and measurement noises. The HPL under the single-fault hypothesis can be determined by projecting the test statistic into the position domain and can be expressed as (Parkinson et al., 1996):

$$
H P L_{f}=\text { Slope }_{\text {max }} \cdot \text { pbias }_{\mathrm{B}}
$$




$$
\text { Slope }_{\max }=\max _{i} \text { Slope }_{i}=\max _{i}\left(\sqrt{\frac{\boldsymbol{A}_{1 i}^{2}+\boldsymbol{A}_{2 i}^{2}}{\boldsymbol{S}_{i i}}}\right)
$$

where Slope $_{i}$ is the sensitivity of the horizontal position error to the ith measurement bias (Feng et al., 2009), pbias $_{\mathrm{B}}$ is the minimum detection bias and can be calculated using the method described in Brown (Brown 1998). $\boldsymbol{A}_{i j}$ is the ith row and $j$ th column of matrix $\boldsymbol{A}=\left(\boldsymbol{G}^{\mathrm{T}} \boldsymbol{G}\right)^{-1} \boldsymbol{G}^{\mathrm{T}}$ and $\boldsymbol{S}_{i i}$ denotes the ith diagonal element of the matrix $\boldsymbol{S}=\boldsymbol{I}-\boldsymbol{G A}$. In the traditional RAIM algorithm, it is assumed that the fault induced positioning error is accounted for the major part of total system error. A study by Diesel et al. (1995) pointed out that one also needs to consider the effect of measurement noises while developing the statistic for the total system error. This is known as the horizontal uncertainty level (HUL) and is expressed as follows:

$$
\begin{gathered}
H U L=K_{\mathrm{H}} \sigma_{\mathrm{H}} \\
\sigma_{\mathrm{H}}^{2}=\boldsymbol{P}_{11}+\boldsymbol{P}_{22}+2 \boldsymbol{P}_{12}
\end{gathered}
$$

where $K_{\mathrm{H}}$ is the factor that derived from the probability of missed detection. It should be noted that the formulation of $\sigma_{\mathrm{H}}$ is due to the correlation of the noises in the east and north directions, which can be directly obtained from matrix $\boldsymbol{P}_{k}$ in the filtering process.

Three different methods can be employed to achieve a conservative HPL for the integrated GPS/DR/DEM system aided by map-matching. The first method is based on the AIME proposed by Diesel et al. (1995) in which the equal weight is assigned to the two components (i.e. $\mathrm{HPL}_{f}$ and HUL) of the HPL as shown below:

$$
H P L_{\mathrm{AIME}}=\sqrt{H P L_{f}^{2}+H U L^{2}}
$$

The second method assumes that the measurement fault is perfectly correlated with the measurement noise resulting in the generation of a worst-case HPL that can be expressed as follows:

$$
H P L_{\text {worst-case }}=H P L_{f}+H U L
$$

The worst-case HPL is widely used in the solution separation method proposed by Honeywell (Brenner 1996). It has been revealed by Lee et al. (Lee and O’Laughlin 2000) that the HPL estimated from the solution separation method is generally larger than that of the AIME making the solution separation based HPL more difficult to obtain sufficient availability. This is because the solution separation based HPL is greatly affected by the userto-satellite geometry and the number of satellites in view, especially during the RAIM holes (i.e. the number of available satellites is less than four). In the urban area, the RAIM holes become more frequent as a result of the problems associated with the availability of GPS signal.

The third method assumes that the measurement fault is independent with the measurement noise and yields: 


$$
H P L_{\text {independent }}=\max \left(H P L_{f}, H U L\right)
$$

The third method is also termed as the snapshot method. One of the drawbacks in the snapshot approach is the assumption that there is no correlation among the errors in three components (east, north, and height). With the application of the extended Kalman filter, the correlation between different position errors can however be characterised. This is very essential for improving the RAIM performance.

Another disadvantage of the traditional snapshot RAIM is that this is based on the independent hypothesis and assumes that the measurement noise induced position error is negligible. This kind of assumption is valid for some non-safety-critical application such as non-precision approach (NPA) in the case of aircraft navigation. This is a challenging issue for the land vehicle navigation systems with more integrity risk resources.

It is obvious from the above discussion that $H P L_{\text {independent }} \leq H P L_{\text {AIME }} \leq H P L_{\text {worst-case }}$. This means that $H P L_{\text {worst-case }}$ is too conservative to satisfy the stringent availability requirement for the mission and safety-critical applications, while $H P L_{\text {independent }}$ ignores the correlation between the measurement noises and the measurement bias and is inaccurate to express the upper bound of total system error. It is found that the effect of correlation could induce the difficulty in satisfying the integrity risk requirement in terms of slow ramp errors ( $\mathrm{Li}$ et al., 2011). The AIME based HPL could provide the edge in dealing with the effect of slow ramp errors (Bhatti 2007).

In order to compensate the effect of the correlation on the HPL, the sigma inflation can be applied to the HUL which is a combination of the AIME and the worst-case based method in essence. The ratio between the covariance $\sigma_{\text {cor }}$ for the correlation and $\sigma_{\mathrm{H}}$ is defined as the sigma inflation factor $\alpha$ and is expressed as:

$$
\alpha=\frac{\sigma_{\text {cor }}}{\sigma_{\mathrm{H}}}
$$

It can be anticipated that the sigma inflation may cause a negative impact on the HPL performance. The HPL loss can however be manipulated by properly selecting the sigma inflation factor. The sigma inflation based HPL can be expressed as:

$$
H P L_{\text {inf }}=\sqrt{H P L_{f}^{2}+\left(1+\alpha^{2}\right) H U L^{2}}
$$

It is obvious that $H P L_{\text {inf }} \geq H P L_{\text {independent }}$ is satisfied. However it is necessary that

$$
0 \leq \alpha^{2} \leq \frac{2 H P L_{f}}{H U L}
$$

should be satisfied such that $H P L_{\text {inf }} \leq H P L_{\text {worst-case }}$.

The allocated integrity risk requirement is the primary criterion for the selecting the sigma inflation factor. According to Khanafseh (2009), the integrity risk can be expressed as:

$$
\mathrm{I}_{\text {req }}=\mathrm{P}\left(\left|\tilde{\boldsymbol{x}}_{\mathrm{H}}\right| \geq H P L_{\text {inf }}\right)=\int_{-\infty}^{H P L_{\text {inf }}} f\left(\tilde{\boldsymbol{x}}_{\mathrm{H}}\right) \mathrm{d} \tilde{\boldsymbol{x}}_{\mathrm{H}}+\int_{H P L_{\text {inf }}}^{+\infty} f\left(\tilde{\boldsymbol{x}}_{\mathrm{H}}\right) \mathrm{d} \tilde{\boldsymbol{x}}_{\mathrm{H}}
$$


As horizontal position estimation error $\tilde{\boldsymbol{x}}_{\mathrm{H}} \sim \mathrm{N}\left(\mu_{\mathrm{H}}, \sigma_{\mathrm{H}}^{2}\right)$, where $\mu_{\mathrm{H}}$ is the bias in the position error and can be obtained with the use of the solution separation method, the above equation can be rewritten as:

$$
\mathrm{I}_{\text {req }}=\Phi\left(\frac{-H P L_{\mathrm{inf}}-\mu_{\mathrm{H}}}{\sigma_{\mathrm{H}}}\right)+1-\Phi\left(\frac{H P L_{\mathrm{inf}}-\mu_{\mathrm{H}}}{\sigma_{\mathrm{H}}}\right)
$$

By combining Eq. (25) and Eq. (22), the optimal inflation factor can be achieved with according to minimize the integrity risk ultimately. In order to obtain the optimal inflation factor an iterative searching algorithm as discussed in can be used over the range (as shown in Eq. (23)) to enhance the searching efficiency.

\subsection{Fault detection}

According to Quddus et al. (2006), the integrity of a map matching algorithm can be defined as its ability to correctly identify a link and to accurately determine the vehicle location on the link for a particular epoch. In this paper, we assume that the failures related to integrity may arise from either GPS raw measurements or incorrect link identification in the map-matching process.

The extended Kalman filter based test statistics for the fault detection can be expressed as:

$$
t_{k}=\frac{1}{n}\left\|\boldsymbol{r}_{k}\right\|_{\boldsymbol{C}_{k}^{-1}}=\frac{\sqrt{\boldsymbol{r}_{k}^{\mathrm{T}} \boldsymbol{C}_{k}^{-1} \boldsymbol{r}_{k}}}{n}
$$

where $\boldsymbol{r}_{k}$ is the innovation and $\boldsymbol{C}_{k}$ represent the variance of the innovation, which can be calculated as:

$$
\begin{gathered}
\boldsymbol{r}_{k}=\boldsymbol{y}_{k}-\hat{\boldsymbol{y}}_{k \mid k-1} \\
\boldsymbol{C}_{k}=\boldsymbol{H}_{k} \boldsymbol{P}_{k, k-1}^{-1} \boldsymbol{H}_{k}^{\mathrm{T}}+\boldsymbol{R}_{k}
\end{gathered}
$$

The detection threshold $T$ can be achieved through the probability of false alarm.

$$
\mathrm{P}_{\mathrm{fa}}=\mathrm{P}\left(t_{k}>T \mid \boldsymbol{b}=0\right)=\frac{1}{M} \int_{T}^{\infty} f_{\chi^{2}(n)}(s) \mathrm{d} s
$$

In the traditional RAIM scheme, $M=1$ indicates a constant false alarm rate. One of the disadvantages of the constant false alarm rate is that it is difficult to detect the fault related to small-bias fault arising from the map-matching result. The first map-matching point may cause a domino effect on the subsequent positioning points on the candidate link because of the application of link length constraint. Besides the consistency check proposed by Velaga et al. (2010), the fault detection threshold should be more stringent and conservative. In such a case $M$ should be equal to the number of candidate links meaning that the fault detection would be more stringent to detect any possible small-bias fault as the detection threshold is decreased. It can be anticipated that with the application of variable false alarm rate, the fault detection performance can be enhanced with the cost of availability loss. 


\section{Experiment and Validation}

In order to reliably evaluate and validate the developed algorithm, one needs to employ representative datasets from all operational environments including rural, suburban and urban settings. Therefore, data were collected from two real-world experiments: (1) one from Nottingham (3,363 epochs) and (2) central London (2,399 epochs). Data from Nottingham represent typical rural and suburban environments while data from London represent a typical harsh operational environment (e.g. Zhao et al. 2003). A single-frequency Ublox GPS receiver (AEK-6T) was used to collect raw GPS measurements. The heading and displacement measurements were collected from another Ublox receiver (AEK-4R). Road network data employed in the analysis was the UK's Integrated Transport Network database obtained from the UK Ordnance Survey. Network data have common features that include node attributes (e.g. node ID and geographic coordinates) and link attributes (e.g. link ID, start node, end node and length). Network data also contain additional features such as grade separation that is employed to determine whether links are physically connected and the direction of traffic flow that is used to identify the start node and the end node of a link. The same network data were employed in both study areas.

Two tightly-coupled carrier phase GPS receivers integrated with a high-grade inertial navigation system were employed to collect reference data: (1) a NovAtel SPAN for the Nottingham test and an iMar for the central London test. These integrated systems are capable of providing the true vehicle trajectory at the centimetre level (assuming that the period of maximum continuous GPS outage is within the operating range of the high-grade inertial navigation systems). The map-matching result and integrity monitoring performance are analysed for both datasets.

The input parameters for the integrity monitoring are set up as follows (Feng and Ochieng 2007): the false alarm rate is taken as $6.67 \times 10^{-4}$, the missed detection rate is 0.001 , the integrity risk is $2 \times 10^{-7}$ and the horizontal alarm limit for the availability level is $50 \mathrm{~m}$. The sample frequency for the raw GPS pseudorange data is $1 \mathrm{~Hz}$. For the Nottingham dataset that includes a suburban area, the positioning threshold $T H_{m m}$ for map-matching is assumed as $30 \mathrm{~m}$ which is the maximum positioning error. The weight $w^{\mathrm{PD}}$ is set up as 0.5. The autocorrelation time constants $\tau_{h}$ and $\tau_{t}$ are taken as 150s and 30s. For the London dataset, due to the worse positioning accuracy, the positioning threshold for map-matching is assumed to be $100 \mathrm{~m}, w^{\mathrm{PD}}$ is set up as 0.45 , and the auto-correlation time constants $\tau_{h}$ and $\tau_{t}$ are set up as 50 s and 30s.

\subsection{Results for the Nottingham Dataset}

The Nottingham is selected to be typical location for the suburban area, where the quality of GPS and DR measurements can be relatively higher than in the urban area. Therefore it will be beneficial to test the best performance of the proposed algorithm in positioning accuracy and map-matching efficiency.

\subsubsection{HPL performance}

The HPL results from the GPS, GPS/DR/DEM, map-matching aided CFAR and the mapmatching aided VFAR with the sigma inflation approach (i.e. the enhanced RAIM) are shown in Fig. 3. As can be seen in Fig.3, there are no RAIM holes in the data and the tightly-coupled 
GPS/DR/DEM has reduced the HPL relative to the stand-alone GPS. The CFAR based HPL has resulted in a relatively low HPL as aided by the map-matching algorithm. The VFAR based HPL is however slightly worse than the CFAR because the VFAR aims at improving the fault detection with the cost of availability loss. The availability loss in the case of VFAR has been mitigated by the application of the proposed sigma inflation method.

\section{INSERT FIGURE 3 HERE}

\subsubsection{Fault detection performance}

Figure 4-a shows that there are some cases of incorrect link identification as a result of failures in the map-matching process. The red points and the blue '+' symbol are the positioning result from the proposed tightly-coupled GPS/DR/DEM integrated navigation algorithm and the map-matched results based on red points, respectively. As can be seen in Fig.4-a, due to the severe measurements noises near junctions, the multiple weights for the position difference and heading difference are not the optimal ones. The applied weightbased topological map-matching algorithm is sensitive to junctions where there are multiple candidate links and causes some misidentification cases, which are specifically marked with red circle and zoomed in the sub-figures. Because the first point on the selected link may cause a domino effect on the following points, the fault detection threshold should be more stringent so as to detect the small-bias fault caused by the incorrect link identification. The improvement in fault detection performance is however achieved with the cost of availability loss as stated above.

\section{INSERT FIGURE 4-a AND 4-b HERE}

The fault detection performance with the application of VFAR is shown in Fig. 4-b. With combing Fig. 4-a and Fig. 4-b and examining the time stamps of misidentifications cases, it can be seen that there are two fault cases from the GPS/DR/DEM measurements. After the map-matching process, the VFAR has found to be capable of detecting more incorrect link identification than the CFAR, which mainly due to the fault detection performance has been enhanced with the use of a stringent detection threshold. It should be noted that the VFAR is also able to detect the fault from the GPS/DR/DEM measurements suggesting that the developed enhanced RAIM algorithm is capable of detecting the faults as a result of a failure either in the GPS/DR/DEM or in the map-matching process for the case of Nottingham dataset. Another should be noted that there is one misidentification case (i.e. the time stamp 2375) in one of junction which is due to the severe measurements noise from DR .

\subsection{Results from the Central London Dataset}

In order to testify the effectiveness of the proposed algorithm in dealing with uncertainties from both the GPS measurements and mismatching from map-matching, an experiment was carried out in central London, where the severer GPS and DR measurement noises and more mismatching cases can be anticipated due to the frequent GPS signal blockages and turnings in the junctions.

\subsubsection{HPL performance}

The HPL results from the GPS, GPS/DR/DEM, the constant false alarm (CFAR) with the aiding of map-matching, and the VFAR for the central London dataset are shown in Fig. 5. 
As can be seen, the map-matching aided CFAR has provided better HPL relative to the standalone GPS and the map-matching aided VFAR. It is noted that the measurement noise is severe in the data collected in the central London and as a result, the HPL is relatively high compared to the Nottingham dataset. This is one of the reasons that a non-Gaussian error distribution should be considered while calculating the system HPL. It can be said that the developed tightly-coupled navigation system provides integrity monitoring during the GPS RAIM holes as there are redundant measurement from multiple navigation sources (i.e. GPS, DR, DEM and historical map-matched results). As enough redundant measurements are obtained from various navigation sources, the developed method has the ability to provide integrity during GPS outages.

\section{INSERT FIGURE 5 HERE}

\subsubsection{Fault detection performance}

The map-matching result for the central London data is shown in Fig. 6-a. As can be seen, the developed map-matching algorithm is sensitive to the turning manoeuvres where there are multiple candidate links. As the heading measurement noises are relatively high when the vehicle makes a turn, most of the link misidentification cases have occurred during such manoeuvres. This illustrates that the fault detection should be more stringent when there are multiple candidate links. With a combination of Fig. 5 and Fig. 6-a, it is noticeable that the tightly-coupled navigation system provide a stable positioning solution even when there are frequent and long-periods (maximum 23seconds) RAIM holes. However, due to the effect of strong measurement noise, which mainly caused by DR output delay effect under high dynamic state and much severe multipath error of GPS measurement in the corner, there are more link misidentifications cases in the urban area with respect to the suburban area because the proposed map-matching is sensitive to the measurments noise. Some sepcific link misidentification cases in the junctions or corners are shown in the red circule of Fig. 6-a. It further indicates the requirment of better fault detection performance in the urban area.

\section{INSERT FIGURE 6-a HERE}

The corresponding fault detection process is shown in Fig.6-b. It is noticeable that the amplitude of the test statistics are much higher in London than Nottongham. It indicates that the measurement noises are relatively high in the urban area due to the effect of urban canyons and frequent turning manoeuvres. It can be seen that the proposed VFAR is able to detect more link misidentifiaction occurred at the junctions than the traditional CFAR. Furthermore, due to the improvement in positioing accuracy as a result of the applicaton of map-matching method, the false alarm cases have reduced compared with the one without the application of map-mathcing.

\section{INSERT FIGURE 6-b HERE}

\subsection{Performance Assessment}

The results on the performance of the developed RAIM algorithm for the Nottingham and London datasets are summarised in Table 1. 
As can be seen in Table 1, the developed map-matching algorithm that employed additional road features improves the positioning accuracy to $2.1 \mathrm{~m}(95 \%)$ for the case of Nottingham data and 11.5m (95\%) for the case of central London data. As expected, there are however more link misidentification cases in the urban area, which means that the developed weight-based map-matching algorithm is sensitive to the measurement noises. Under frequent RAIM holes in the urban area, the availability of the developed low-cost tightly-coupled GPS/DR/DEM system has improved.

Because the measurement noise is relatively high in the urban area, the effect of the nonperfection distribution of measurement noise should be properly taken into account while calculating system HPL using the sigma inflation of measurement noise with the purpose of satisfying the integrity risk requirement.

Based on the post-processing of the experiment results and using the Matlab 'tic' function, it was found that the time required to process 3,363 fixes (i.e. Nottingham data) is 203 seconds and the time required to process 2,399 fixes (i.e. London data) is 597 seconds. This has achieved using a PC with 4GB RAM and 3.4GHz processing speed. This suggests that the developed integrity monitoring method can process 16 positioning fixes per second for the Nottingham data and 4 positioning fixes per second for the London data. As can be noticed, the average time required to process a fix in the London area is longer than that of in the Nottingham area. This is due to the fact that the map-matching algorithm needs to process more links in London than that of in Nottingham as roads are denser in the London area.

\section{Conclusions}

This paper develops a new integrity monitoring algorithm for map-matching by considering uncertainties associated with navigation sensors and link identification simultaneously. This has achieved in three innovative ways: (1) the use of an extended Kalman filter to integrate various navigation sensors and map data in a tightly-coupled fusion approach, (2) the application of the 'variable' false alarm rate ( in contrast to the traditionally employed 'constant' false alarm rate) in monitoring the integrity of map-matching resulting in enhanced performance and (3) since the non-perfect distribution of the measurement noise and the use of the variable false alarm rate reduces the system availability, the sigma inflation for a non-Gaussian distribution of measurement noises was employed in the integrity monitoring to address this issue. This suggests that the developed novel integrity monitoring algorithm is capable of detecting both GPS raw measurements' fault and map-matching failures simultaneously with the aid of variable false alarm rate.

As there are no widely acceptable integrity parameters for safety and mission-critical intelligent transport systems and services, the developed method is capable of satisfying stringent requirements with the false alarm rate with 0.001 and the missed detection rate of $6.67 \times 10^{-4}$. This will have a significant impact as navigation modules of many safety and mission-critical intelligent transport systems such as autonomous and intelligent vehicles can be supported by the developed integrity method. The cost of implementing the developed integrity method would be 'low' as the requirement with respect to the required hardware can be met at low-cost (i.e. a low-cost gyroscope and a single-frequency GPS receiver) without compromising the level of integrity performance. 


\section{Acknowledgement}

The project was funded by a grant from the UK Engineering and Physical Science Research Council (EPSRC; Grant Reference: EP/F018894/1). The authors would like to thank Professor Washington Ochieng and Ioannis Demetriou (both from the Centre for Transport Studies at Imperial College London) and Dr Xiaolin Meng, Dr Chris Hill and Dr Lei Yang (all from IESSG at the University of Nottingham) for their support during the data collection.

\section{References}

Bhatti, U., I., 2007. Improved Integrity Algorithms for Integrated GPS/INS Systems in the Presence of Slowly Growing Errors, PhD Thesis, Imperial College London, UK.

Blanch, J., Walter., T., Enge, P., 2007. Understanding PHMI for Safety of Life Applications in GNSS, In: Proceedings of the 2007 National Technical Meeting of The Institute of Navigation, San Diego, CA, pp. 305-310.

Brenner, M., 1996. Integrated GPS/Inertial Fault Detection Availability, Navigation 43 (2), 111-130.

Brown, R., G., 1998. Solution of the Two-Failure GPS RAIM Problem under Worst-Case Bias Conditions: Parity Space Approach, Navigation 44 (4), 425-432.

Chen, W., Li, Z., Yu, M., Chen, Y., 2005. Effects of Sensor Errors on the Performance of Map Matching. The Journal of Navigation 58 (2), 273-282.

Diesel, J., Luu, S., 1995. GPS/IRS AIME: Calculation of Thresholds and Protection Radius Using Chi-Square Methods. In: Proceedings of the 8th International Technical Meeting of the Satellite Division of The Institute of Navigation, Palm Springs, CA, pp. 1959-1964. Feng, S., Ochieng, W., 2007. Integrity of Navigation System for Road Transport. In Proceedings of 14th ITS World Congress, Beijing, China.

Feng, S., Ochieng, W., Moore, T., Hill, C., Hide, C.,2009. Carrier Phase-Based Integrity Monitoring for High-Accuracy Positioning. GPS Solutions 13 (1), 13-22.

Khanafseh, S., Langel, S., Pervan, B., 2009. H1-Integrity of Carrier Phase Navigation Algorithms Using Multiple Reference Receivers. In: Proceedings of the 2009 International Technical Meeting of The Institute of Navigation, CA, pp: 236-247

Lee, Y. C., O’Laughlin, D., G., 2000. Performance Analysis of A Tightly Coupled GPS/Inertial System for Two Integrity Monitoring Methods. Navigation 47 (3), 175-190. Li, L., Quddus, M., Ison, S., Zhao, L., 2012. Multiple Reference Consistency Check for LAAS: A Novel Position Domain Approach. GPS Solutions 16 (2), 209-220.

Misra, P., Enge, P., 2001. Global Positioning System: Signals, Measurements, and Performance. Ganga-Jamuna Press, Lincoln, Mass.

Ochieng, W., Sauer, K., Walsh, D., Brodin, G., Griffin, S., Denny, M., 2003. GPS Integrity and Potential Impact on Aviation Safety. Journal of Navigation 56 (1), 51-65.

Ober, P. B., 2003. Integrity monitoring and prediction of navigation systems. PhD Thesis. TU Delft.

Parkinson, B., Spilker, J., Axelrad, P., Enge, P.,1996. Global Positioning System: Theory and Applications Volume II. Washington, DC: American Institute of Aeronautics and Astronautics, Inc.

Quddus, M., Ochieng, W., Noland, R., 2006. Integrity of Map-Matching Algorithms. Transportation Research C: Emerging Technologies 14 (4), 283-302.

Quddus, M., Zheng, Y., 2011. Low-Cost Tightly Coupled GPS, Dead-Reckoning, and Digital Elevation Model Land Vehicle Tracking System for Intelligent Transportation Systems. In: the 90th Annual Meeting of the Transportation Research Board, Washington, D.C. 
Velaga, N., Quddus, M., Bristow, A., 2010. Map-Aided Integrity for a Land Transport Navigation System. In: the 89th Annual Meeting of the Transportation Research Board, Washington, D.C.

White, C., Bernstein, D., Kornhauser, A., 2000. Some Map Matching Algorithms for Personal Navigation Assistants. Transportation Research C: Emerging Technologies 8 (1), 91-108.

Zhao, L., Ochineg, W. Y., Quddus, M. A., Noland, R. B., 2003. An Extended Kalman Filter Algorithm for Integration GPS and Low Cost Dead Reckoning System Data for Vehicle Performance and Emissions Monitoring. Journal of Navigation 56(2), 257-275. 


\section{FIGURES}

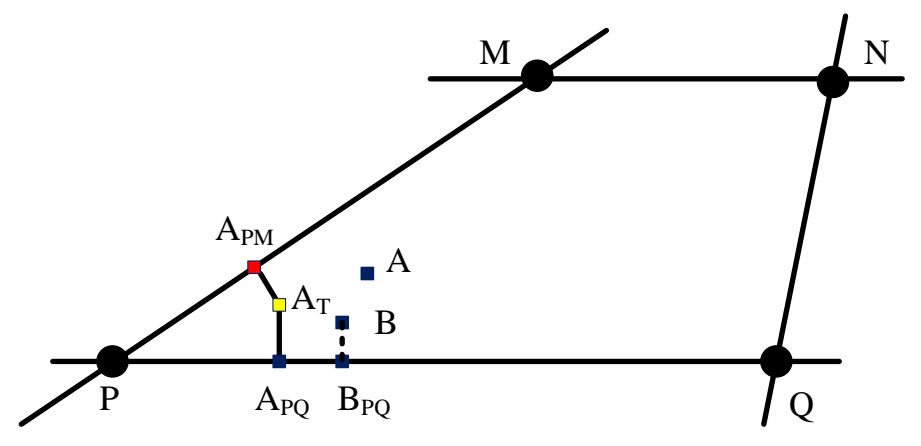

Fig. 1 Map-matching process in the junction 


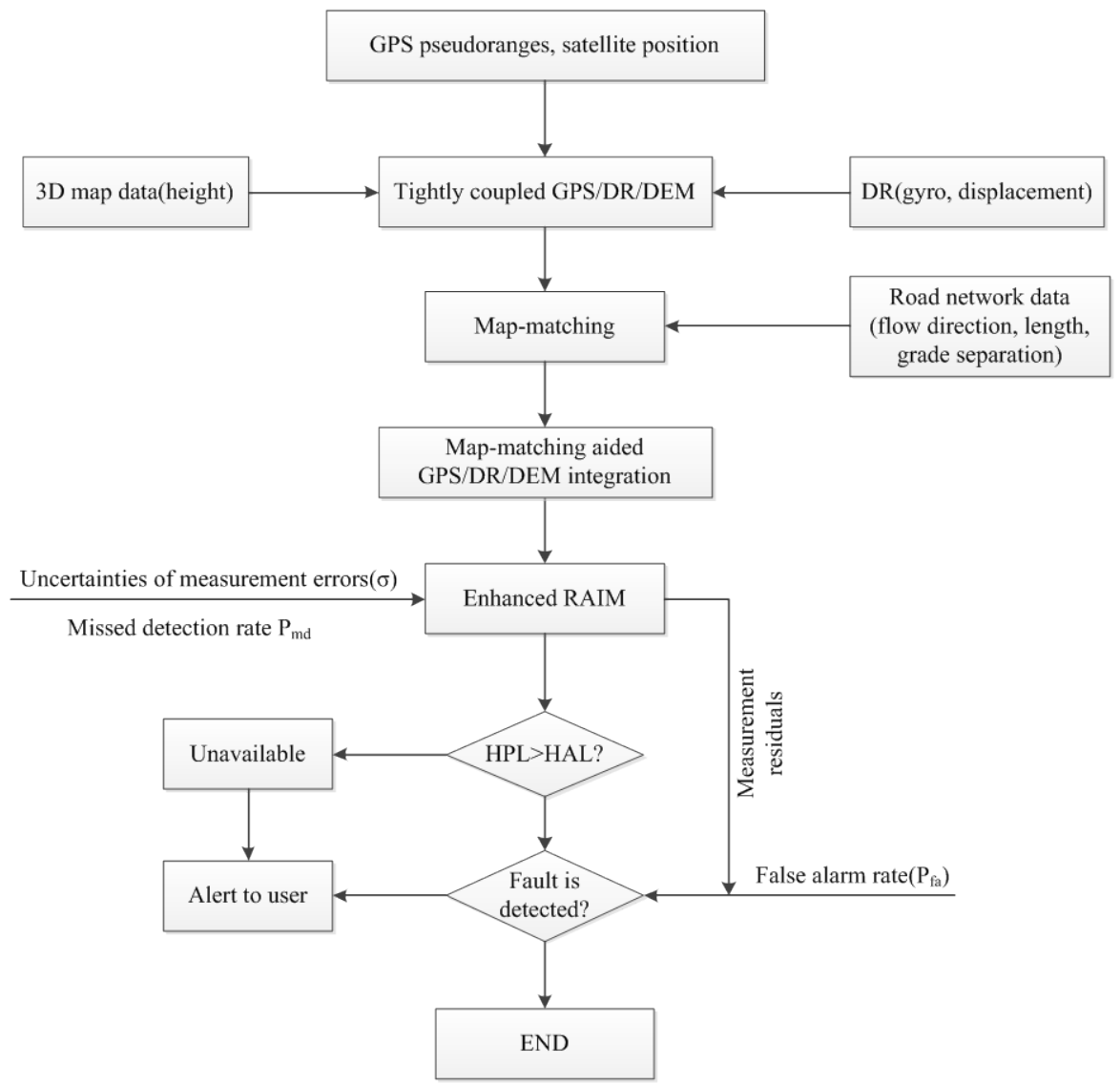

Fig. 2 Flowchart of enhanced RAIM for map-matching integrity 

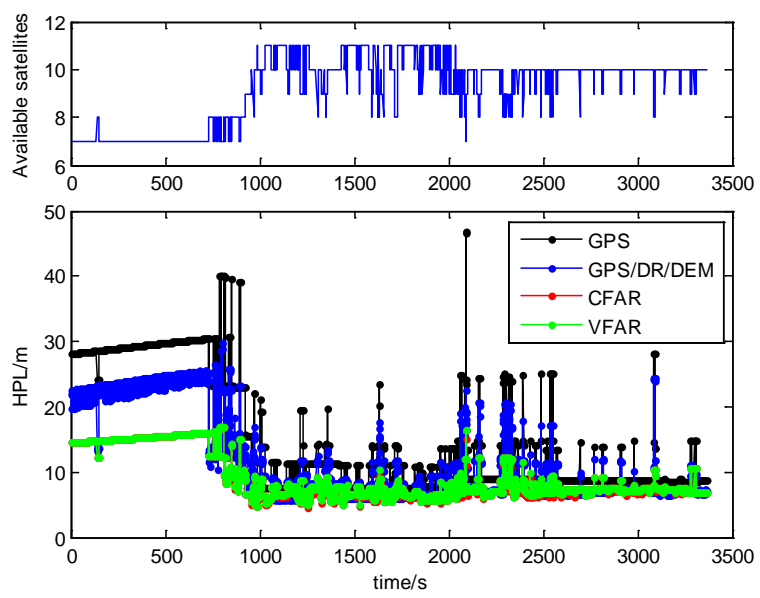

Fig. 3 HPL performance in Nottingham 


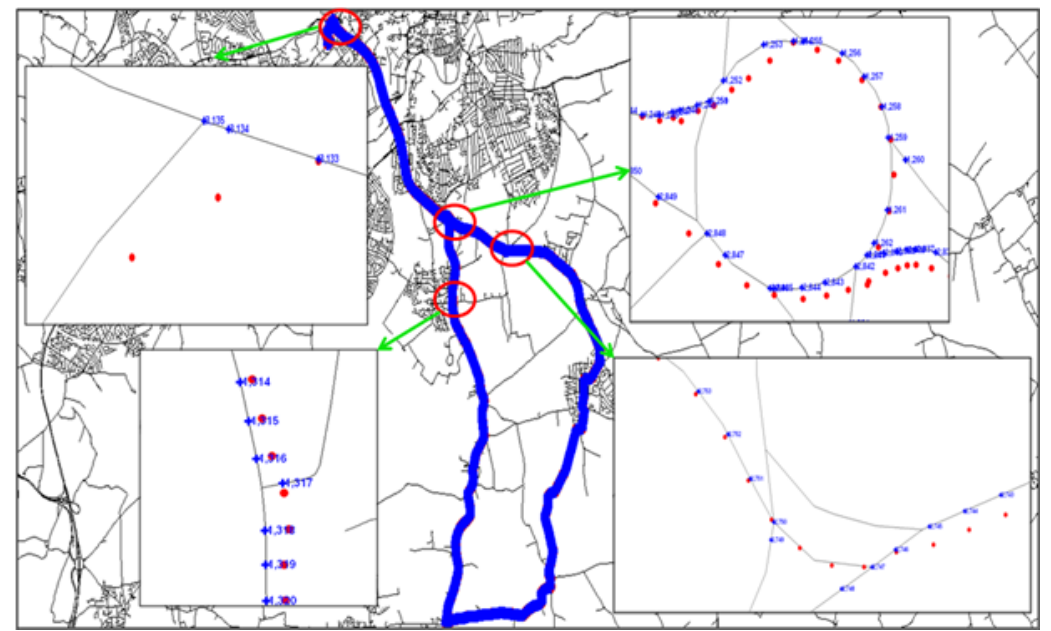

Fig. 4-a Cases with the incorrect link identification (Nottingham data) 

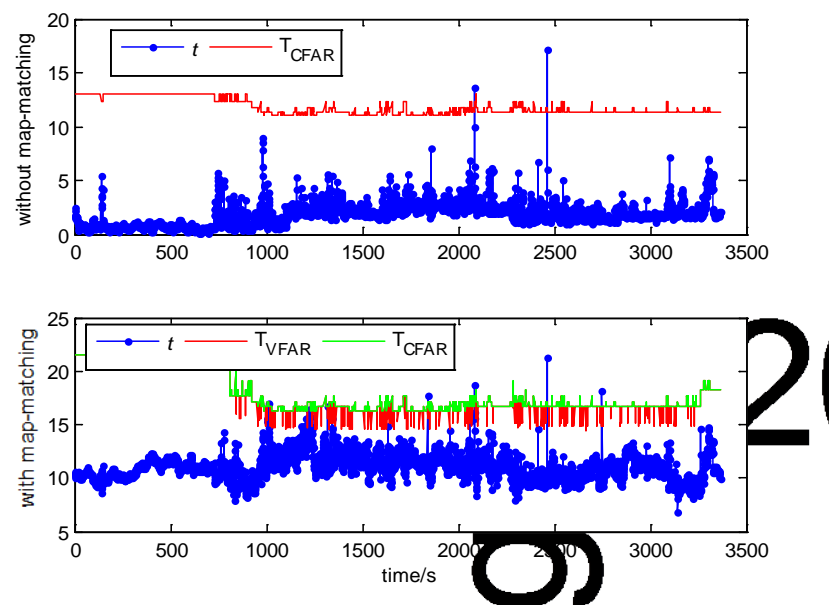

Fig. 4-b Fault detection process for the Nottingham data
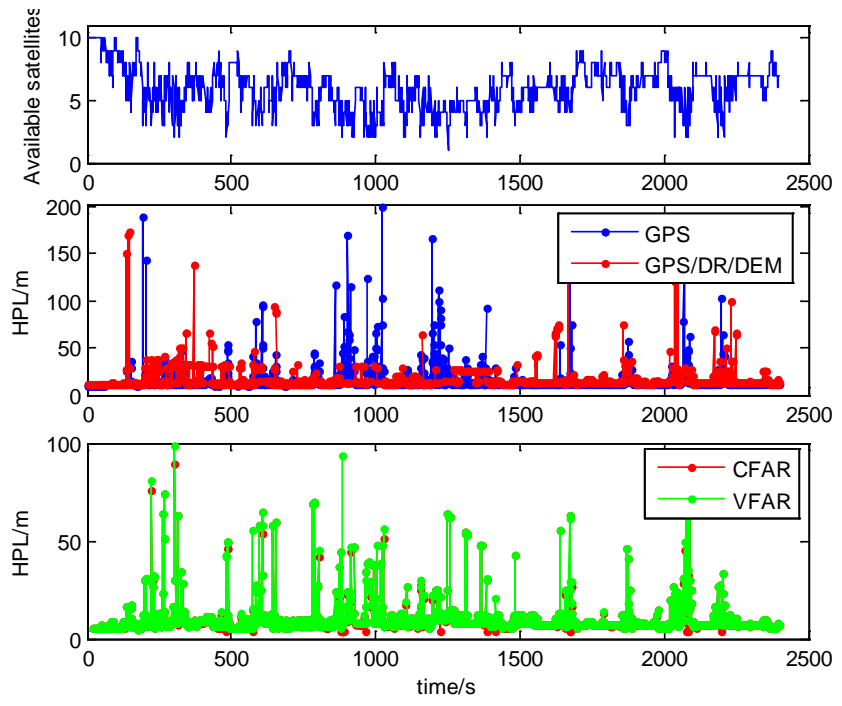

Fig. 5 HPL performance in central London 


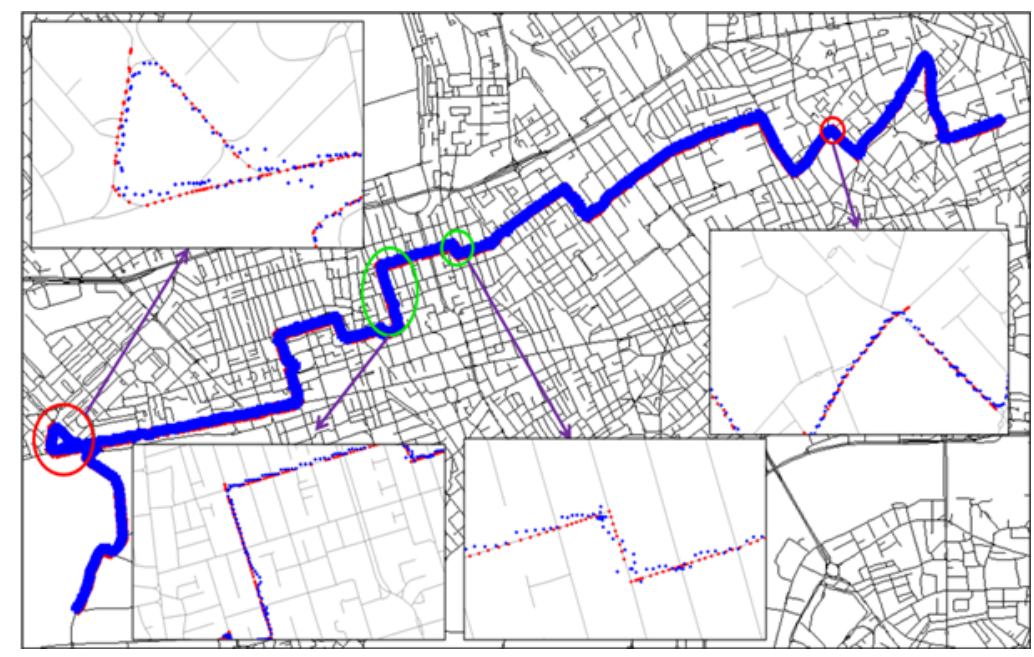

Fig. 6-a Link misindentification cases (central London data)

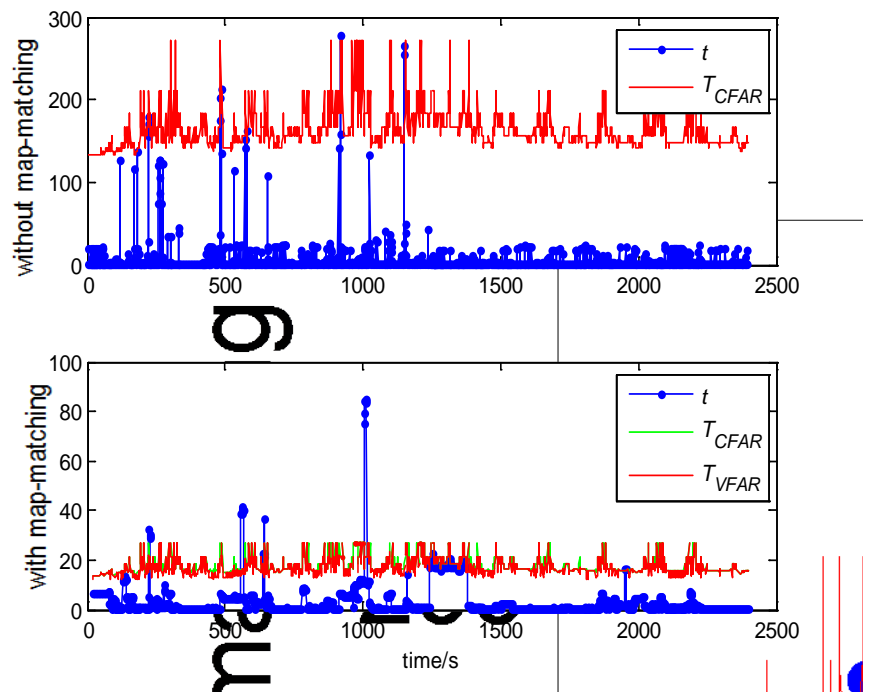

Fig. 6-b Fault detection process corresponding to Fig.6-a 


\section{TABLE}

Table 1 Performance Comparison

\begin{tabular}{|c|c|c|c|c|c|}
\hline & & \multicolumn{2}{|l|}{ Nottingham } & \multicolumn{2}{|c|}{ Central London } \\
\hline & & $\begin{array}{l}\text { without map- } \\
\text { matching }\end{array}$ & $\begin{array}{l}\text { with map- } \\
\text { matching }\end{array}$ & $\begin{array}{l}\text { without map- } \\
\text { matching }\end{array}$ & $\begin{array}{l}\text { with map- } \\
\text { matching }\end{array}$ \\
\hline \multicolumn{2}{|c|}{ 2D accuracy (CEP 95\%, m) } & 2.5 & 2.1 & 15.2 & 11.5 \\
\hline \multicolumn{2}{|l|}{ Availability (\%) } & $100 \%$ & $100 \%$ & $94 \%$ & $97 \%$ \\
\hline \multicolumn{2}{|l|}{ Mismatched cases } & N/A & $99.8 \%$ (6/3363) & N/A & $97.7 \%(53 / 2,399)$ \\
\hline \multicolumn{2}{|l|}{ Missed detection cases } & N/A & 2 & N/A & 11 \\
\hline \multicolumn{2}{|l|}{ False alarm cases } & 2 & 1 & 9 & 3 \\
\hline \multirow{2}{*}{ Correct detection cases } & CFAR & 2 & 1 & 7 & 26 \\
\hline & VFAR & N/A & 5 & N/A & 41 \\
\hline
\end{tabular}

